\title{
Elasticity of rubber-like materials measured by AFM nanoindentation
}

\author{
D. C. Lin $^{1 *}$, E. K. Dimitriadis ${ }^{2}$, F. Horkay $3^{*}$ \\ ${ }^{1}$ Laboratory of Integrative and Medical Biophysics, National Institutes of Health, 9 Memorial Drive, Bldg. 9 Rm. 1E118, \\ Bethesda, MD 20892, USA \\ ${ }^{2}$ National Institute of Biomedical Imaging and Bioengineering, National Institutes of Health, 13 South Drive, \\ Bldg. 13 Rm. 3N17, Bethesda, MD 20892, USA \\ ${ }^{3}$ Laboratory of Integrative and Medical Biophysics, National Institutes of Health, 13 South Drive, Bldg. 13 Rm. 3W16, \\ Bethesda, MD 20892, USA
}

\begin{abstract}
We exploit the force spectroscopy capabilities of the atomic force microscope in characterizing the local elasticity of rubber-like materials. Extraction of elastic properties from force curves usually relies on the linear theory pioneered by Hertz. While the Hertzian force-indentation relationships have been shown to be accurate in modeling the contact mechanics at sufficiently shallow indentation depths, the linear deformation regime of the probed material is exceeded in many practical applications of nanoindentation. In this article, a simple, nonlinear force-indentation equation based on the Mooney-Rivlin model is derived and used to fit data from the indentation of lightly crosslinked poly(vinyl alcohol) gels in equilibrium with water. The extracted values of Young's modulus show good agreement with those obtained by both macroscopic compression testing and by fitting truncated portions of the force curves with the Hertz equation.
\end{abstract}

Keywords: mechanical properties, atomic force microscopy, polymer gels, indentation

\section{Introduction}

Despite its prevalence in various fields as a technique for measuring the local mechanical properties of elastic materials, the accuracy of indentation testing utilizing the atomic force microscope (AFM: see Figure 1) remains equivocal. Aside from artifacts arising from factors that can affect instrument performance (e.g., drift due to temperature variations), the causes of the ambiguity can be methodological (e.g., uncertainties in determining cantilever bending stiffness and tip dimensions) or analytical (e.g., uncertainties in robustness and accuracy of the data processing method) in nature. With care and the employment of existing technologies, environmental and methodological effects can be minimized. However, the latter cate-

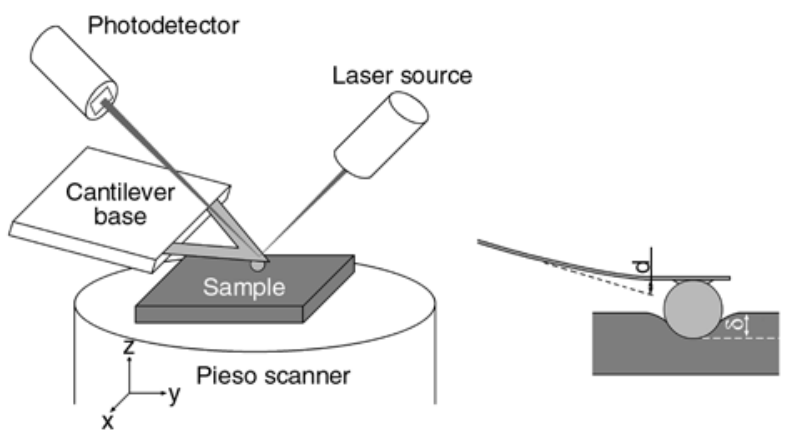

Figure 1. Schematics of the atomic force microscope (left) and indentation of the sample by the tip (right). Displacement control is achieved by moving the sample (as shown) or the cantilever base, usually with piezo tube actuators. Force is inferred from laser-based measurements of cantilever deflection $d$. The indentation depth $\delta$ is dependent on both $d$ and the vertical displacement of the scanner (or the cantilever base) $z$.

*Corresponding authors, e-mail: lindavid@ mail.nih.gov, horkay@ helix.nih.gov (C) BME-PT and GTE 
gory of sources is not as easily addressed because data processing methods are model dependent and no clear performance benchmark exists. Furthermore, indentation at all length scales can deviate significantly from ideal behavior due to tip-sample interactions (e.g., adhesion) and material nonuniformities. This is especially true of gels and other rubber-like materials.

Recently, we developed and validated a robust and comprehensive scheme for extracting Young's moduli from the indentation of soft materials based on linear elastic contact mechanics theory $[1,2]$. For a set of poly(vinyl alcohol) - henceforth abbreviated to PVA - gels at different polymer concentrations, Young's moduli agreed well with macroscopic compression tests when indentation strains did not exceed the linear elastic limit. These results are consistent with the generally accepted view that small-strain deformation of many rubber-like materials is virtually a linear elastic process and can be modeled accordingly. Depending on the magnitude of tip-sample interactions, however, it is oftentimes difficult to control the indentation depth of each stroke of the AFM probe. Furthermore, tip-sample interactions often result in decreased signal-tonoise ratios. The effect is usually most pronounced in the vicinity of the tip-sample contact point; under such circumstances, accuracy is adversely affected by limiting the analysis to data points that do not exceed a strain threshold. For materials that exhibit rubber elastic behavior, derivation of a single contact mechanics equation relating force and indentation depth is therefore necessary. Such a model would also be applicable in estimating the large-strain mechanical response of soft biological materials such as cells and tissues, where the use of linear elasticity theory generally leads to significant errors in Young's modulus [3]. In this work, we propose an approximate relationship suitable for the indentation of Mooney-Rivlin materials with spherical probes and test it by fitting to data obtained from the AFM indentation of highly swollen PVA gels. Accuracy of this model is assessed by comparison with results from macroscopic compression tests and from fitting initial, small-strain portions of each dataset with the classical Hertz equation.

\section{Theory}

The indentation problem is based on the Hertz theory of contact between two elastic spheres in the absence of adhesion [4]. Johnson et al. [5] pioneered the theory of adhesive contact, citing evidence of deviation from Hertzian behavior in numerous experiments as the motivation for developing the now well-known Johnson-KendallRoberts (JKR) theory. Other contributors to the field include Derjaguin et al. [6], Tabor [7], and Maugis [8]. For the purposes of this work, only the Derjaguin-Muller-Toporov (DMT) theory is considered. The JKR and DMT theories were found by Tabor to apply to opposite extremes of the relationship between surface force and sample compliance, with the DMT theory pertaining to relatively stiff samples and weak adhesive forces [7].

The Hertz and DMT equations ((1) and (2)) for the indentation of a linear elastic, infinite half-space with a rigid sphere are $[4,6]$ :

$$
\begin{aligned}
& F=F_{n}+F_{a d}=\frac{4 E R^{1 / 2} \delta^{3 / 2}}{3\left(1-v^{2}\right)} \\
& a=\sqrt{R \delta}
\end{aligned}
$$

where $F$ is the net indentation force, $F_{n}$ is the applied normal force, $F_{a d}$ is the tip-sample adhesive force ( $=0$ in the Hertz theory), $\delta$ is the indentation depth, $a$ is the contact radius, $R$ is the radius of the sphere, and $E$ and $v$ are Young's modulus and Poisson's ratio of the indented sample, respectively. In the DMT theory, the constant adhesive force is related to the interfacial energy $(\gamma)$ by Equation (3):

$F_{a d}=2 \pi \gamma R$

According to Yoffe [9], Hertz stated explicitly that Equation (2) applies only to cases in which the contact radius is small relative to the radius of the indenter (i. e., $a / R<0.1$ ). In practice, however, contact radii frequently exceed the imposed limit. Experimental and analytical support for applying the equation at large relative contact radii can be found in tests performed by Kumar and Narasimhan [10] and the theoretical studies of Yoffe [9]. In the macroscopic indentation of polymethyl methacrylate samples using stainless steel balls, Kumar and Narasimhan found excellent agreement between measured values of the contact radius and those predicted by Equation (2) for $a / R$ 
approaching 0.14. Yoffe developed a first-order correction to the Hertzian contact radius at large indentation depths and demonstrated that the deviation from the Hertz theory as the contact area widened was dependent on $v$. As $v$ approached the incompressibility limit of 0.5 , it was found that Equation (2) began to over predict the magnitude of the contact radius. For example, when $v=0.4$, the error in $a$ predicted by Equation (2) increased from $1 \%$ at $a / R \sim 0.28$ to $6 \%$ at $a / R \sim 0.53$. These errors can be considered acceptably small, even at the large strains that are frequently applied in the indentation of polymer gels.

\section{Materials and methods}

\subsection{Sample preparation and testing}

PVA solutions were prepared by dissolving PVA (molecular weight $70000-100000$; Sigma) in deionized water at $99^{\circ} \mathrm{C}$ to make a stock solution with a concentration of $14 \%(w / w)$. Gels were made by crosslinking the PVA solution with glutaraldehyde at $\mathrm{pH} \sim 1.5$ (adjusted by addition of $\mathrm{HCl}$ ). To prepare gel samples at two different levels of compliance, the PVA concentration was changed while maintaining a constant crosslink density (one unit of GDA per 100 units of vinyl alcohol). The elastic moduli of these gels differed by roughly an order of magnitude corresponding to the typical range of biological soft tissues such as cartilage. Gel cylinders and thick layers $(2 \mathrm{~mm}$ to $>1 \mathrm{~cm}$ ) with final PVA concentrations of $6 \%$ and $12 \%$ $(\mathrm{w} / \mathrm{w})$ were cast in molds $(1 \mathrm{~cm}$ in diameter and $1 \mathrm{~cm}$ in height) and $35 \mathrm{~mm}$ Petri dishes, respectively. All samples were stored in deionized water until testing.

A bench top materials testing system (Stable Micro Systems, UK) was used to perform displacementcontrolled compression of the gel cylinders at a ramp speed of $1 \mathrm{~mm} / \mathrm{s}$. The undeformed dimensions of each cylinder were measured prior to compression with a micrometer. The Young's moduli of the PVA gels were calculated from the neoHookean equation (4):

$\sigma=G\left(\lambda-\lambda^{-2}\right)$

where $\sigma$ is the engineering stress, $\lambda$ is the compression ratio, and $G$ is the shear modulus. In the experiment, $\lambda$ was varied in the range $0.6<\lambda<1$. The absence of volume change and barreling during the compression measurements was checked [1].

Nanoindentation of gels was performed using a commercial AFM (Bioscope I with a Nanoscope IIIA controller, Veeco Instruments, Santa Barbara, CA) seated atop an inverted optical microscope. General-purpose silicon nitride tips were used (model DNP, Veeco). The cantilevers were modified by gluing either a $9.6 \mu \mathrm{m}$ diameter polystyrene bead or a $5.5 \mu \mathrm{m}$ diameter glass bead near the tip. The spring constant of each cantilever was determined using the thermal tune method [11]. Multiple force curves for each PVA film were collected using the 'force-volume' mode of the AFM. In this automated raster scanning method, the user defines the size of the square region to be scanned, the resolution, and the relative trigger threshold (i. e., the maximum cantilever deflection). Because the gels were assumed to be relatively homogeneous, the resolution was set to the lowest limit of $16 \times 16$ indentations covering a $20 \mu \mathrm{m} \times 20 \mu \mathrm{m}$ region. Relative trigger thresholds were set to either 100 or $50 \mathrm{~nm}$.

\subsection{Processing of AFM indentation data}

An algorithm that we developed previously [1, 2] was used as the basis for automated processing of indentation data. Briefly, the contact point dependent method requires the identification of multiple reference points, shown in Figure 2 for both adhesive and non-adhesive analysis. These points are used to transform the typical raw values of cantilever deflection $(d)$ and base displacement $(z)$ to applied force and indentation, respectively. The conversions are given by Equations (5), (6) and (7):

$F_{n}=k_{c}\left(d-d_{0}\right) ;$ negligible adhesion

$F_{n}=k_{c}\left(d-d_{1}\right) ;$ significant adhesion

$$
\begin{aligned}
\delta= & \left(z-z_{0}\right)-\left(d-d_{0}\right) \\
& =(z-d)-\left(z_{0}-d_{0}\right)=w-w_{0}
\end{aligned}
$$

where $k_{c}$ is the spring constant of the cantilever, $\left(z_{0}, d_{0}\right)$ are the coordinates of the contact point, and $\left(z_{1}, d_{1}\right)$ are the coordinates of the point of zero applied force (see Figure 2). The transformed variable $w=z-d$ is introduced for simplification. The adhesive force is then related to the reference points by Equation (8): 


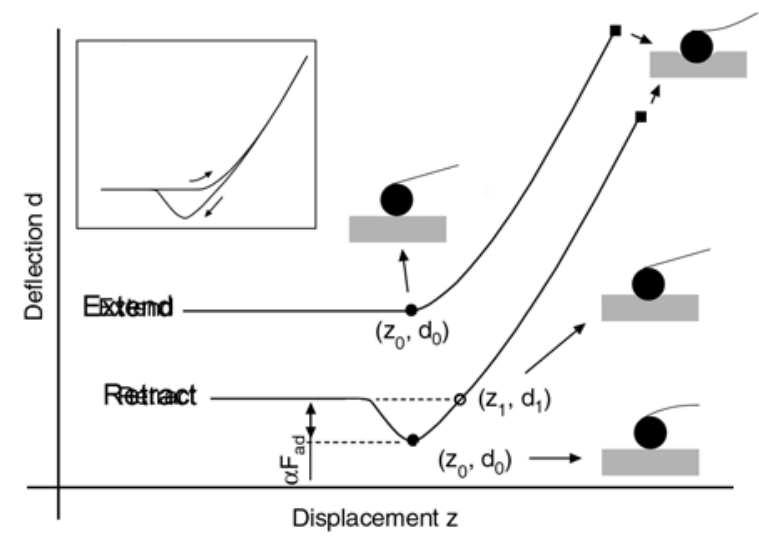

Figure 2. A typical set of deflection-displacement curves in extension and retraction with negligible adhesive interactions in extension and significant adhesion upon retraction. The two curves are offset from each other for clarity; they are plotted without offset in the inset. In extension, only the contact point $\left(z_{0}, d_{0}\right)$ is required to transform the data to force $v s$. indentation. In retraction, two reference points are needed - the contact point and the point of zero applied force $\left(z_{1}, d_{1}\right)$. The schematics show the bending of the cantilever at the reference points and at the point of maximum indentation.

$F_{a d}=k_{c}\left(d_{1}-d_{0}\right)$

Substitution of Equations (5) through (8) into Equation (1) recasts the force-indentation relation into a form appropriate for fitting the raw data and extracting the values of $E$.

\section{Results and discussion}

In this section, we first develop a nonlinear contact mechanics model that describes the force-indentation relationship for rubber-like materials. The derivation is based on the concepts discussed in the theoretical section and on the Mooney-Rivlin formalism. A comparison is then made between the predictions of this model and the experimental data obtained for the PVA hydrogels.

\subsection{Formulation of a nonlinear contact mechanics model}

The Hertz and DMT equations are based on the theory of linear elasticity and therefore subject to its inherent limitations, including the requirement of geometric (i. e., stress-strain) linearity. As previously mentioned, it may not always be feasible to limit indentation depths to the linear regime. In the realm of rubber elasticity, many phenomenological theories have been developed. One of the simplest and perhaps most well-known of the stress-strain relations derived from these theories is the Mooney-Rivlin equation (9) [12]:

$\sigma=2 C_{1}\left(\lambda-\lambda^{-2}\right)+2 C_{2}\left(\lambda-\lambda^{-3}\right)$

where $\sigma$ is the stress, $\lambda$ is the extension ratio, and $C_{1}$ and $C_{2}$ are constants. We wish to use Equation (9) as the basis of a force-indentation relationship. To that end, we define the effective or average stress and strain due to indentation. The indentation stress, $\sigma^{*}$, can be set equal to the mean contact pressure (force over the contact area), given by Equation (10):

$$
\sigma^{*}=\frac{F}{\pi a^{2}}
$$

The indentation strain, $\varepsilon^{*}$, is defined by Equation (11) [13]:

$$
\varepsilon^{*}=\frac{a}{R}
$$

For linear elastic (Hertzian) contact, it can be seen that $\sigma^{*}$ and $\varepsilon^{*}$ are linearly proportional, giving a Hookean equation (12) of the form:

$$
\sigma^{*}=\zeta \varepsilon^{*}, \text { with } \zeta=\frac{4 E}{3 \pi\left(1-v^{2}\right)}
$$

In order to derive force-indentation relations for materials obeying the constitutive Equation (9), disparities in sign conventions for stress and strain between Equations (9) and (12) must first be resolved. In Equation (9), standard engineering convention applies, with stresses and strains positive in tension. This implies that $\lambda>1$ in tension and $\lambda<1$ in compression. Because indentation is viewed as a compressive process, the sign convention for $\sigma^{*}$ and $\varepsilon^{*}$ are opposite from the standard convention. Replacing $\sigma$ with $-\sigma^{*}$ and $\lambda$ with $\left(1-\varepsilon^{*}\right)$ in Equation (9) yields Equation (13):

$$
\begin{aligned}
\frac{\sigma^{*}}{\varepsilon^{*}}= & B_{1}\left(\frac{\varepsilon^{* 3}-3 \varepsilon^{* 2}+3 \varepsilon^{*}}{\varepsilon^{* 3}-2 \varepsilon^{* 2}+\varepsilon^{*}}\right)+ \\
& B_{2}\left(\frac{\varepsilon^{* 3}-3 \varepsilon^{* 2}+3 \varepsilon^{*}}{-\varepsilon^{* 4}+3 \varepsilon^{* 3}-3 \varepsilon^{* 2}+\varepsilon^{*}}\right)
\end{aligned}
$$


where the constants $C_{1}$ and $C_{2}$ no longer hold the same meaning and have been replaced with $B_{1} / 2$ and $B_{2} / 2$, respectively.

Substitution of Equations (10) and (11) into Equation (13) results in a relationship between indentation force and contact radius. To obtain a more practicable equation in terms of force and indentation, the relationship between contact radius and indentation is required. Assuming that the manner in which $a$ varies with $\delta$ is independent of material linearity, we use Equation (2) as a first approximation. Algebraic manipulation yields Equation (14):

$$
\begin{aligned}
F= & \pi R^{1 / 2} B_{1}\left(\frac{\delta^{5 / 2}-3 R^{1 / 2} \delta^{2}+3 R \delta^{3 / 2}}{\delta-2 R^{1 / 2} \delta^{1 / 2}+R}\right)+ \\
& \pi R^{1 / 2} B_{2}\left(\frac{R^{1 / 2} \delta^{5 / 2}-3 R \delta^{2}+3 R^{3 / 2} \delta^{3 / 2}}{-\delta^{3 / 2}+3 R^{1 / 2} \delta-3 R \delta^{1 / 2}+R^{3 / 2}}\right)
\end{aligned}
$$

where $F=F_{a d}+F_{n}$ when adhesive interactions are present. At infinitesimal strain or indentation depth, Equation (1) applies, and Equation (14) reduces to Equation (15):

$$
B_{1}+B_{2}=\frac{4 E_{0}}{9 \pi\left(1-v^{2}\right)}
$$

where $E_{0}$ is the initial Young's modulus and $v=0.5$ for incompressible materials. Equations (14) and (15) comprise an approximate Mooney-Rivlin contact mechanics model that can be applied to the indentation of rubber-like materials; elastic properties of the samples are represented by the extracted Young's moduli. The model reduces to the neoHookean form when $B_{2}=0$.

Justification for the assumption that the contact radius is independent of material linearity can be found in the results of the finite element analyses performed by Mesarovic and Fleck [14] on the indentation behavior of elastic-plastic solids. Such materials undergo strain hardening in compression, as do Mooney-Rivlin solids. Under uniaxial tension, the Ramberg-Osgood hardening relationship employed in the finite element study has the form of Equation (16):

$$
\frac{\varepsilon}{\varepsilon_{0}}=\frac{\sigma}{\sigma_{0}}+\left(\frac{\sigma}{\sigma_{0}}\right)^{m}
$$

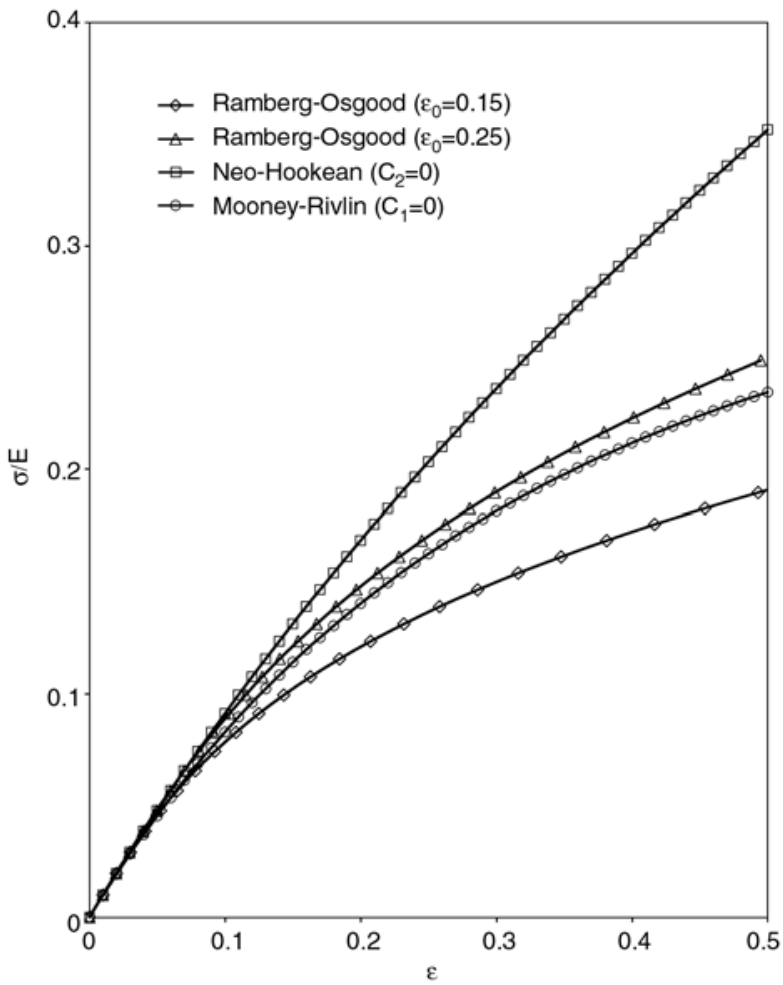

Figure 3. Comparison of the Ramberg-Osgood elastic-plastic hardening equation and the Mooney-Rivlin equation in tension. Normalized engineering stress-strain curves, where $\sigma$ is stress, $E$ is Young's modulus, and $\varepsilon$ is strain, are shown for different cases of the two relationships. Following Mesarovic and Fleck, a hardening exponent of 3 is used in the Ramberg-Osgood equation [14]. Yield strains $\left(\varepsilon_{0}\right)$ of $15 \%$ and $25 \%$ are shown. The two extreme cases of the MooneyRivlin equation, given by Equation (9), are plotted: $C_{2}=0$ (representative of a neo-Hookean solid) and $C_{1}=0$.

where $\varepsilon_{0}$ and $\sigma_{0}$ are the strain and stress at the yield point, respectively. The strain-hardening exponent $m$ defines the deformation behavior, with $m=1$ representing linear elastic response and $m \rightarrow \infty$ corresponding to elastic-ideally plastic response; an intermediate value of $m=3$ was used by Mesarovic and Fleck in their study. The mathematical similarity between the Mooney-Rivlin and RambergOsgood equations is demonstrated in Figure 3, where stress-strain curves are shown for a typical hardening material with linear Young's modulus of $100 \mathrm{kPa}$ and a linear strain limit of approximately $15 \%$. Mesarovic and Fleck found that the contact radius followed the form of Equation (2) beyond the yield point; with further indentation, contact radii predicted by Equation (2) became increasingly smaller than the actual values. This likely due to the 
Table 1. Young's moduli of PVA gels from compression and AFM indentation (mean \pm SD)

\begin{tabular}{|c|c|c|c|c|c|}
\hline \multirow{2}{*}{$\%$} & \multirow{2}{*}{ Macro. [kPa] } & \multicolumn{2}{|c|}{ Small strain, linear elastic } & \multicolumn{2}{c|}{ Large strain, Mooney-Rivlin } \\
\cline { 3 - 6 } & & Extend [kPa] & Retract [kPa] & Extend [kPa] & Retract [kPa] \\
\hline 6 & $21.51 \pm 0.59$ & $16.55 \pm 2.74$ & $19.39 \pm 3.26$ & $18.23 \pm 2.38$ & $19.51 \pm 4.69$ \\
\hline 12 & $115.50 \pm 1.86$ & $113.66 \pm 6.06$ & $108.98 \pm 9.17$ & $115.82 \pm 7.21$ & $110.08 \pm 13.17$ \\
\hline
\end{tabular}

permanent pile-up of material around the indenter, which is typically associated with the indentation of elastic-plastic materials such as metals $[15,16]$.

\subsection{Comparison of the model with experi- mental results}

Results of the macroscopic compression and AFM indentation tests are summarized in Table 1. There is generally good agreement between the macroscopic Young's moduli and values obtained from the small strain and large strain analyses of the AFM indentation data. Adhesion during indentation was evident only in the retraction strokes, prior to tip-sample separation. The small strain analysis was performed by truncating the datasets at an indentation strain of about $15 \%$ and applying Equation (1). The large strain analysis using Equation (14) was performed without data truncation.
In fitting the macroscopic compression data with Equation (9) over a large range of $\lambda(0.6<\lambda<1)$, it was found that $C_{2} \sim 0$ within the experimental error. This is consistent with the macroscopic, neoHookean behavior of PVA gels tested under similar conditions [17,18] and of lightly crosslinked, highly swollen gels in general $[12,19]$. Values of the Mooney-Rivlin fitting parameters $B_{1}$ and $B_{2}$ from fitting of the indentation data with Equation (14) are summarized in Table 2. At the microscopic length scale probed by the AFM, the large variability in $B_{2}$ may reflect inhomogeneities due

Table 2. Mooney-Rivlin fitting coefficients for large strain analysis (mean \pm SD)

\begin{tabular}{|r|c|c|c|c|}
\hline \multirow{2}{*}{$\%$} & \multicolumn{2}{|c|}{ Extend } & \multicolumn{2}{c|}{ Retract } \\
\cline { 2 - 5 } & $\mathbf{B}_{\mathbf{1}}[\mathbf{k P a}]$ & $\mathbf{B}_{2}[\mathbf{k P a}]$ & $\mathbf{B}_{\mathbf{1}}[\mathbf{k P a}]$ & $\mathbf{B}_{\mathbf{2}}[\mathbf{k P a}]$ \\
\hline 6 & $4.92 \pm 0.97$ & $-1.49 \pm 0.55$ & $8.08 \pm 2.28$ & $-4.09 \pm 1.49$ \\
\hline 12 & $39.60 \pm 4.46$ & $-17.76 \pm 3.15$ & $43.48 \pm 10.20$ & $-21.50 \pm 7.32$ \\
\hline
\end{tabular}

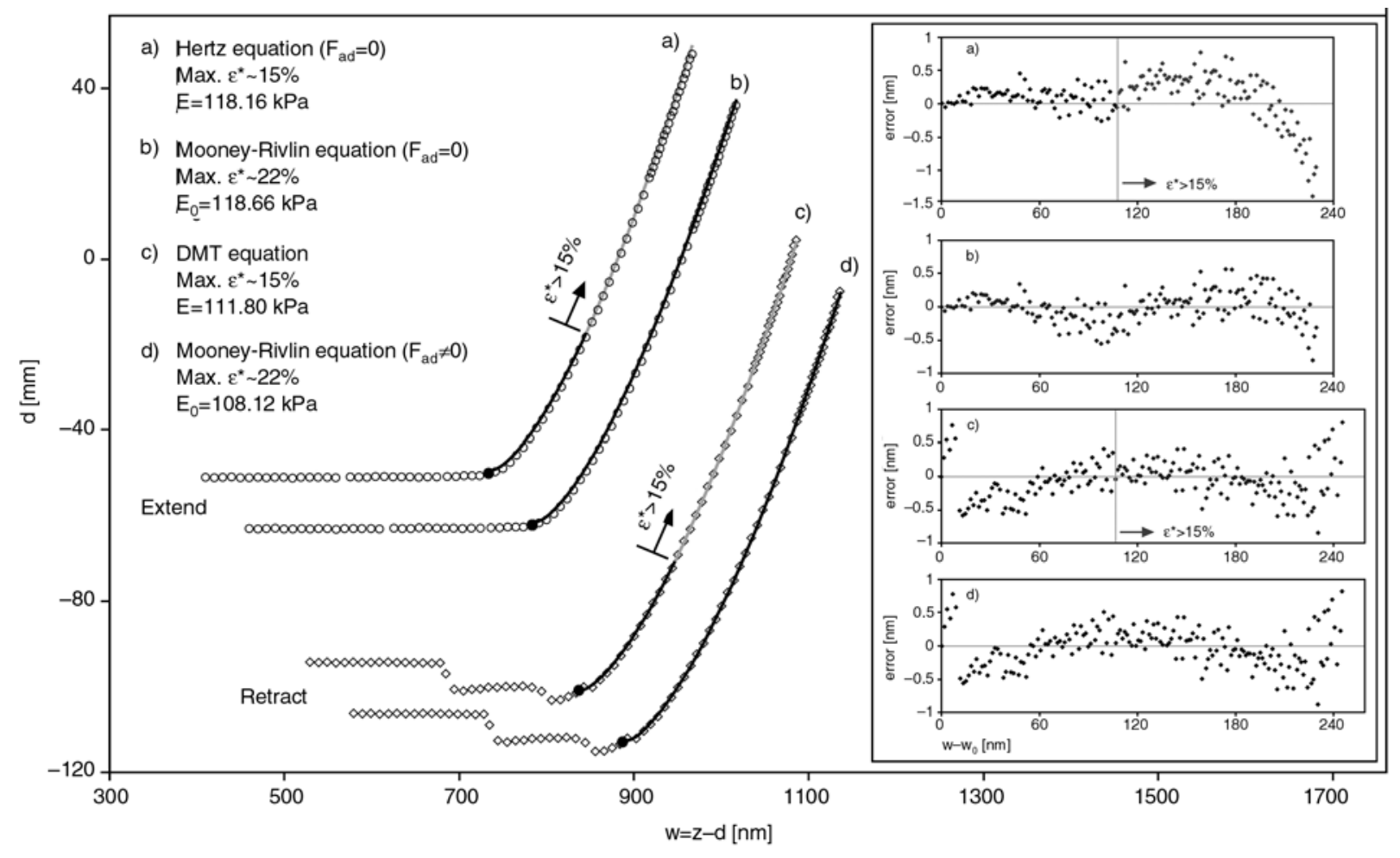

Figure 4. Sample dataset from the indentation of the $12 \%$ gel. $d$ is the cantilever deflection and $z$ is the base displacement. The extension and retraction curves (every fifth point is plotted) are offset from one another and each is shown with the small strain linear elastic fit and the large strain Mooney-Rivlin fit. The linear elastic fits have been extended beyond $15 \%$ strain for comparison (extended portions are shown in gray). Plots of the residual errors for each fit are displayed in the inset. 


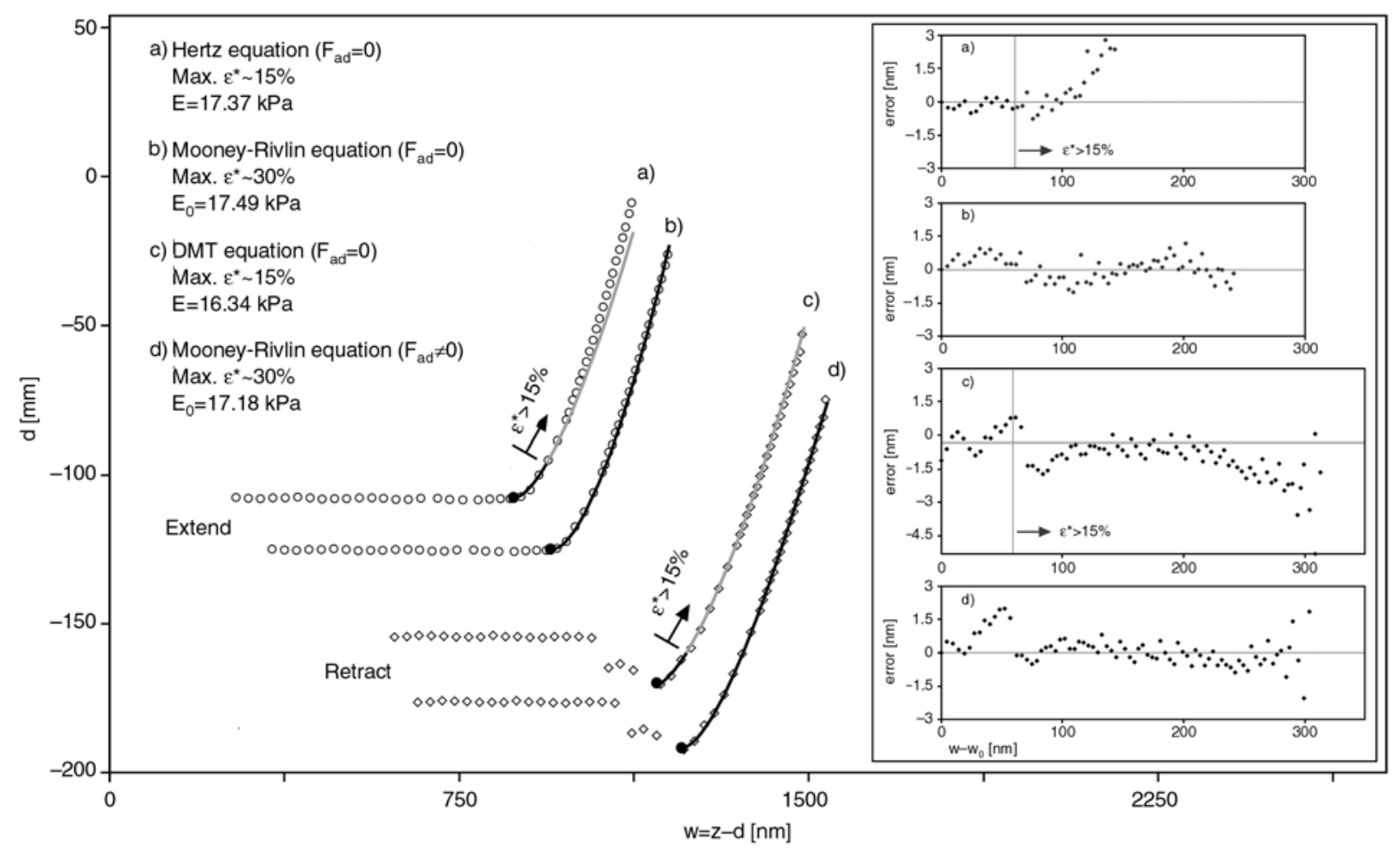

Figure 5. Sample dataset from the indentation of the $6 \%$ gel. $d$ is the cantilever deflection and $z$ is the base displacement. The extension and retraction curves (every fifth point is plotted) are offset from one another and each is shown with the small strain linear elastic fit and the large strain Mooney-Rivlin fit. The linear elastic fits have been extended beyond 15\% strain for comparison (extended portions are shown in gray). Plots of the residual errors for each fit are displayed in the inset.

likely to local differences in polymer concentration. Equation (14) therefore allows us to detect these structural nonuniformities and quantifies the local deviation from neo-Hookean behavior. It should be mentioned that the Mooney-Rivlin formalism is not a constitutive material law since the values of the material constants may depend on the mode of deformation. Hence, extreme caution should be exercised when applying Equations (9) and (14).

Strains at maximum indentation depth were approximately $22 \%$ and $30 \%$ for the $12 \%$ and $6 \%$ gels, respectively. The $12 \%$ gel exhibited a linear regime that was significantly wider than that of the $6 \%$ gel, as illustrated by the examples in Figures 4 and 5. In fact, the residual errors shown in Figure 4 indicate that the $12 \%$ gel behaved linearly up to the maximum indentation depth. Strain hardening in the $6 \%$ gel is manifested in the poor fit of Equation (1) to the data beyond $\sim 15 \%$ strain, as shown in Figure 5. Regardless of the degree of nonlinearity at maximum indentation depth, Equation (14) was found to be a good fit of both extension and retraction data. Compared to the small strain, linear analysis using Equation (1), the large strain analy- sis using Equation (14) yielded smaller differences between Young's moduli in extension and retraction. We attribute the improved agreement to the inclusion of more data points in the large strain analysis.

Synthetic gels prepared by the crosslinking of polymer chains in solution can be considered isotropic and homogeneous at macroscopic length scales. However, at the submicron level probed by the AFM, spatial variations in mechanical properties due to local concentration nonuniformities become detectable. In biological materials, such variations are much more pronounced, even over regions as small as a few tens of square nanometers. AFM nanoindentation has therefore become a powerful technique for generating elasticity maps of living cells [20-25] and the extracellular matrix [26-31]. To illustrate the differences between relatively homogeneous synthetic polymer gels and biological tissues, Young's modulus maps from $6 \%$ and $12 \%$ PVA gels and from a region of mouse articular cartilage are shown in Figure 6. Over regions of comparable size, local Young's moduli of the PVA gels varied over a much narrower range (approxi- 
$\mathrm{E}[\mathrm{kPa}]$

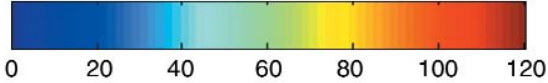

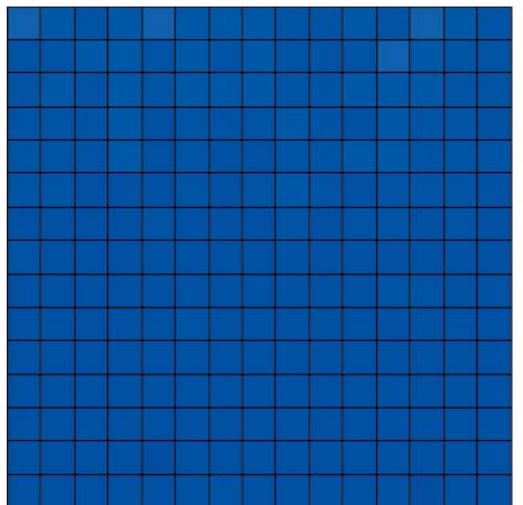

a)

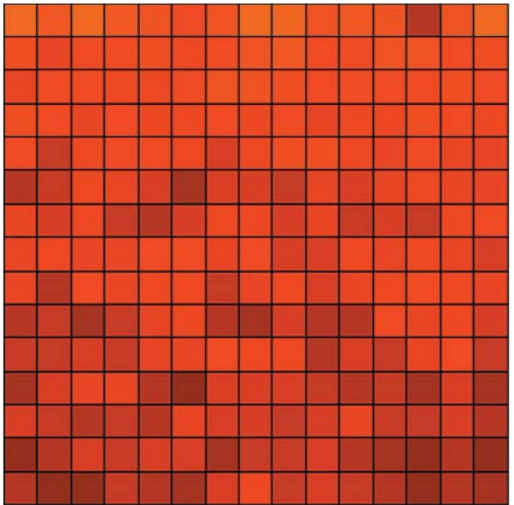

b)

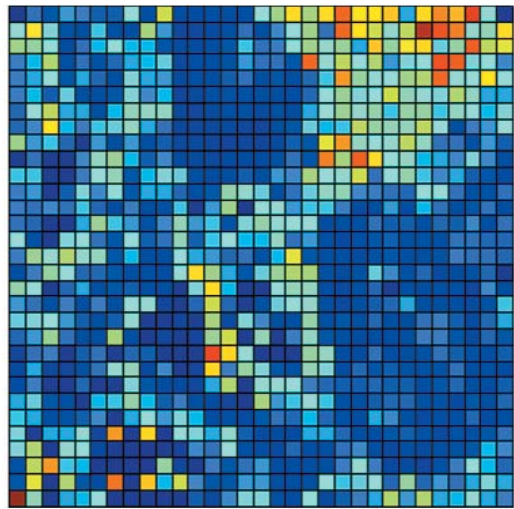

c)

Figure 6. Sample Young's modulus $(E)$ maps from the 'force-volume' indentation of (a) a 6\% PVA gel probed using a $5.5 \mu \mathrm{m}$ glass bead over a $20 \mu \mathrm{m} \times 20 \mu \mathrm{m}$ region at a resolution of $16 \times 16$, (b) a $12 \%$ PVA gel probed using a $9.6 \mu \mathrm{m}$ polystyrene bead over a $20 \mu \mathrm{m} \times 20 \mu \mathrm{m}$ region at a resolution of $16 \times 16$ and, (c) a one-day old mouse articular cartilage sample of approximately $60 \mu \mathrm{m}$ thickness probed using a $5 \mu \mathrm{m}$ polystyrene bead over a $30 \mu \mathrm{m} \times 30 \mu \mathrm{m}$ region at a resolution of $32 \times 32$. The shapes of the force-indentation curves were similar in all cases. Identical color maps are used to compare the degree of inhomogeneity between the samples. The soft regions in the cartilage $(E<25 \mathrm{kPa})$ indicate the presence of several cells.

mately 16 to $24 \mathrm{kPa}$ for the $6 \%$ gel and 90 to $120 \mathrm{kPa}$ for the $12 \%$ gel) than in the cartilage $(<1$ to $120 \mathrm{kPa}$ ). The variability in stiffness in the cartilage corresponds to a high degree of local inhomogeneity, which is a characteristic feature of many biological tissues.

\section{Conclusions}

In rubber-like materials, measurements of elastic moduli using the AFM have been mostly limited to indentation in the linear stress-strain regime. While small strain nanoindentation is practicable in many situations, excessive noise in the vicinity of the contact point can hinder and even preclude its accuracy in other cases. Hence, an easily implemented, large strain contact mechanics model is desirable. The Mooney-Rivlin force-indentation relationship introduced here satisfies this criterion and was shown to be accurate in modeling the indentation behavior of swollen PVA gels. In the AFM probing of any material that exhibits rubber elasticity, it can be applied without the need to limit indentation strains to the linear regime.

\section{Acknowledgements}

This work was supported by the Intramural Research Program of the National Institutes of Health/National Institute of Child Health and Human Development.

\section{References}

[1] Lin D. C., Dimitriadis E. K., Horkay F.: Robust strategies for automated AFM force curve analysis - I. Nonadhesive indentation of soft, inhomogeneous materials. Journal of Biomechanical Engineering, 129, 430 440 (2007).

[2] Lin D. C., Dimitriadis E. K., Horkay F.: Robust strategies for automated AFM force curve analysis - II. Adhesion-influenced indentation of soft, elastic materials. Journal of Biomechanical Engineering, in press (2007).

[3] Costa K. D., Yin F. C. P.: Analysis of indentation: implications for measuring mechanical properties with atomic force microscopy. Journal of Biomechanical Engineering, 121, 462-471 (1999).

[4] Johnson K. L.: Contact mechanics. Cambridge University Press, Cambridge (1985).

[5] Johnson K. L., Kendall K., Roberts A. D.: Surface energy and the contact of elastic solids. Proceedings of the Royal Society of London, Series A: Mathematical, Physical and Engineering Sciences, 324, 301-313 (1971).

[6] Derjaguin B. V., Muller V. M., Toporov Y. P.: Effect of contact deformations on the adhesion of particles. Journal of Colloid and Interface Science, 53, 314-326 (1975). 
[7] Tabor D.: Surface forces and surface interactions. Journal of Colloid and Interface Science, 58, 2-13 (1976).

[8] Maugis D.: Adhesion of spheres: The JKR-DMT transition using a Dugdale model. Journal of Colloid and Interface Science, 150, 243-269 (1992).

[9] Yoffe E. H.: Modified Hertz theory for spherical indentation. Philosophical Magazine, 50, 813-828 (1984).

[10] Kumar M. V., Narasimhan R.: Analysis of spherical indentation of linear viscoelastic materials. Current Science, 87, 1088-1095 (2004).

[11] Burnham N. A., Chen X., Hodges C. S., Matei G. A., Thoreson E. J., Roberts C. J., Davies M. C., Tendler S. J. B.: Comparison of calibration methods for atomicforce microscopy cantilevers. Nanotechnology, 14, $1-6(2003)$.

[12] Treloar L. R. G.: The Physics of Rubber Elasticity. Oxford University Press, Oxford (1975).

[13] Lee K. S., Wuttiphan S., Hu X., Lee S. K., Lawn B. R.: Contact-induced transverse fractures in brittle layers on soft substrates: a study on silicon nitride bilayers. Journal of the American Ceramics Society, 81, 571-580 (1998).

[14] Mesarovic S. D. J., Fleck N. A.: Spherical indentation of elastic-plastic solids. Proceedings of the Royal Society of London, Series A: Mathematical, Physical and Engineering Sciences, 455, 2707-2728 (1999).

[15] Bolshakov A., Pharr G. M.: Influences of pile-up on the measurement of mechanical properties by load and depth sensing indentation techniques. Journal of Materials Research, 13, 1049-1058 (1998).

[16] McElhaney K. W., Vlassak J. J., Nix W. D.: Determination of indenter tip geometry and indentation contact area for depth-sensing indentation experiments. Journal of Materials Research, 13, 1300-1306 (1998).

[17] Horkay F., Nagy M.: Elasticity of swollen polyvinyl alcohol and poly(vinyl acetate) networks. Polymer Bulletin, 3, 457-463 (1980).

[18] McKenna G. B., Horkay F.: Effects of crosslinks on the thermodynamics of poly(vinyl alcohol) hydrogels. Polymer, 35, 5737-5742 (1994).

[19] Dusek K., Prins W.: Structure and elasticity of noncrystalline polymer networks. Advances in Polymer Science, 6, 1-102 (1969).

[20] A-Hassan E., Heinz W. F., Antonik M. D., D'Costa N. P., Nageswaran S., Schoenenberger C., Hoh J. H.: Relative microelastic mapping of living cells by atomic force microscopy. Biophysical Journal, 74, 1564-1578 (1998).
[21] Almqvist N., Bhatia R., Primbs G., Desai N., Banerjee S., Lal R.: Elasticity and adhesion force mapping reveals real-time clustering of growth factor receptors and associated changes in local cellular rheological properties. Biophysical Journal, 86, 1753-1762 (2004).

[22] Domke J., Dannohl S., Parak W. J., Müller O., Aicher W. K., Radmacher M.: Substrate dependent differences in morphology and elasticity of living osteoblasts investigated by atomic force microscopy. Colloids and Surfaces B: Biointerfaces, 19, 367-379 (2000).

[23] Haga H., Sasaki S., Kawabata K., Ito E., Ushiki T., Sambongi T.: Elasticity mapping of living fibroblasts by AFM and immunofluorescence observation of the cytoskeleton. Ultramicroscopy, 82, 253-258 (2000).

[24] Hofmann U. G., Rotsch C., Parak W. J., Radmacher M.: Investigating the cytoskeleton of chicken cardiocytes with the atomic force microscope. Journal of Structural Biology, 119, 84-91 (1997).

[25] Touhami A., Nysten B., Dufrene Y. F.: Nanoscale mapping of the elasticity of microbial cells by atomic force microscopy. Langmuir, 19, 4539-4543 (2003).

[26] Allen D. M., Mao J. J.: Heterogeneous nanostructural and nanoelastic properties of pericellular and interterritorial matrices of chondrocytes by atomic force microscopy. Journal of Structural Biology, 145, 196204 (2004).

[27] Hu K., Radhakrishnan P., Patel R. V., Mao J. J.: Regional structural and viscoelastic properties of fibrocartilage upon dynamic nanoindentation of the articular condyle. Journal of Structural Biology, 136, 46-52 (2001).

[28] Patel R. V., Mao J. J.: Microstructural and elastic properties of the extracellular matrices of the superficial zone of neonatal articular cartilage by atomic force microscopy. Frontiers in Bioscience, 8, 18-25 (2003)

[29] Radhakrishnan P., Lewis N. T., Mao J. J.: Zone-specific micromechanical properties of the extracellular matrices of growth plate cartilage. Annals of Biomedical Engineering, 32, 284-291 (2004).

[30] Stolz M., Raiteri R., Daniels A. U., VanLandingham M. R., Baschong W., Aebi U.: Dynamic elastic modulus of porcine articular cartilage determined at two different levels of tissue organization by indentation-type atomic force microscopy. Biophysical Journal, 86, 3269-3283 (2004).

[31] Tomkoria S., Patel R. V., Mao J. J.: Heterogeneous nanomechanical properties of superficial and zonal regions of articular cartilage of the rabbit proximal radius condyle by atomic force microscopy. Medical Engineering and Physics, 26, 815-822 (2004). 